\title{
CHALLENGES IN MALARIA VECTOR CONTROL; IS THE USE OF DDT THE BEST OPTION?
}

\section{INTRODUCTION}

Malaria is an infection caused by a protozoan parasite of the genus Plasmodium; four species of which infect human beings, the most common being Plasmodium vivax and most deadly being Plasmodium falciparum. The Anopheles mosquito serves as Plasmodium's delivery system, or vector. Only female mosquitoes can transmit it since males don't take blood meals. Anopheles mosquitoes bite mainly during night time hours and early hours in the morning (IDRC, 2008).

\section{HISTORICAL BACKGROUND}

Malaria ranks among the three major health and developmental challenges facing most of the poorest countries in the tropical and sub-tropical regions of the world Tanzania being the one. As early as $2700 \mathrm{BC}$, a disease marked by high fever and an enlarged spleen was noted in ancient China as reported in ancient Chinese documents. Believing the disease was transmitted through the air, the sixteenth-century Italians called the disorder mal'aria (bad or evil air). Plasmodium vivax stowed away with the English going to Jamestown, while Plasmodium falciparum rode along with slaves from Africa.

In 1958 the World Health Organization (WHO) decided to wage a global campaign against malaria and sent workers into South American, African, and Asian villages to spray them with Dichlorodiphenyltrichloroethane (DDT). But except for areas such as Egypt and Southern Europe where the parasite had not been firmly established, in the end the spraying only succeeded in making the mosquitoes pesticide-resistant. By the 1960s, the WHO had scaled its Global Eradication of Malaria Program to one of worldwide malaria control.

\section{PLASMODIUM'S LIFE CYCLE}

The female Anopheles mosquito ingests the reproductive stage of the parasite when it feeds on blood from someone who already has malaria. The parasites then incubate in the insect's mid-gut for a week or more, maturing until they reach an infective form, and are reintroduced into humans when the mosquito feeds again on blood of noninfected persons. A biting mosquito transfers about 10 percent of its parasite load into the victim's capillaries or the tissue around the blood vessels. The parasites make their way to the person's liver in less than 30 minutes of entering the bloodstream and penetrate the liver cells (hepatocytes). During the period when the parasites are maturing further in the liver, as little as eight days or as long as several months the infected person does not feel ill. Two kinds of malaria, P. vivax and P. ovale, can relapse, and some parasites can remain dormant in the liver from several months to four years after a person is infected. Once the parasites leave the liver and re-enter the bloodstream, they invade and multiply in the red blood cells, periodically bursting the cells. Their further development leads to the formation of gametocytes (the parasite's sexual stages), which are picked up and transmitted to others when another mosquito feeds on blood from the infected person. 


\section{PREVENTION AND TREATMENT MEASURE}

Malaria for so long has been prevented through encouraging sanitary improvement (such as filling and draining areas of impounding water) that will result in permanent elimination or reduction of anopheline breeding habitats, larvicides and biological control larvivorous fish, although very rare in our country.

Also night spraying of screened living and sleeping quarters with a liquid or aerosol preparation of pyrethrum or other insecticides. E.g. Rungu insecticides have been intensively emphasized.

Apart from that, in areas where malaria is endemic, installation of screens and use of bed nets has been encouraged where the effectiveness of bed nets is greatly enhanced by impregnation with a synthetic pyrethroid (e.g. permethrin) in hand with periodic intake of chemoprophylaxis.

Finally, the Prompt and Effective Treatment of Acute and Chronic Cases is An Important element of Malaria Control, Artemisinin Combination Therapies (ACTs) are now recommended for treatment of malaria after most of antimalarias drugs in many countries to show resistance. These lifesaving medications are becoming more widely available throughout the world. In January of this year, WHO took stringent measures to help prevent future resistance to antimalarial medicines by banning the use of malaria monotherapy and introduce the effective treatment of malaria by gametocides.

\section{CONTROL MEASURES}

For many years now control of malaria especially elimination of malaria vectors has been an issue of concern due to the skyrocketing number of infant and maternal mortality in developing countries and various methods have been taken since 1958 where WHO first announced the global campaign against malaria through the use of indoor Residual Spraying specifically (DDT) due to its effectiveness and cost effectiveness in application.

Control of malaria has been debated for so many years since 1940s and 1950s where indoor residual spraying (IRS) especially DDT was discussed and seem to be useful in controlling mosquitoes which are the main vectors of the diseases and host in the secondary stage of the development of parasites i.e. Plasmodium. Mosquito has been of target due to the reason that the primary host harbours them in the liver where no chemical action can be effective.

In 2006 WHO announced once again the use of DDT as IRS to be used as the intervention to fight the disease vector after being banned for about 23 years from 1973. But they have been conflicting positions on the advantages and disadvantages of using DDT for malaria control. Some argue that use of DDT will save lives by reducing the number of malaria-related deaths while others argue that the long term exposure to DDT has greater negative effects. There is therefore an information gap on the use of DDT that needs to be filled and a consensus on the issue has not yet been reached.

\section{MEANING OF DDT}

Dichlorodiphenyltrichloroethane (DDT) is a persistent insecticide, meaning that it is only very slowly degraded by natural processes and survives for a long time after its initial application. DDT can also be transported long distances through the atmosphere. Studies in the 1960s revealed that DDT bioaccumulated in the fatty tissue of fish, birds, and animals, and that the DDT levels increased in moving from species to species up the food chain. 


\section{RISKS OF USING DDT}

Individuals involved in spraying DDT have contracted rashes or irritation of the eyes, nose, and throat. Acute exposure at high doses primarily affects the nervous system. Longer-term exposure to DDT may also affect the liver. In low doses, DDT may alter the ability of the liver to metabolize other compounds, while at higher doses, it may cause unusual growths or tumors or the death of whole groups of cells (necrosis).

Children who are breast-fed are at special risk of exposure to DDT, because DDT is ubiquitous and found in human milk in higher concentrations than in cow's milk or other food. People more susceptible to the toxic effects of DDT are individuals with diseases of the nervous system, liver, or blood.

DDT can stay in the environment for more than 30 years and small amounts remaining in soil may be transferred to crops grown there. In human fatty tissue, DDT's half-life is about seven to eight years.

\section{WHY NOT THE USE OF DDT?}

The aim of using DDT is to eradicate mosquitoes; but this primary goal has shown to fail since its first use during 1960s as researches indicates. This research indicates that when DDT spraying is ended, malaria's incidence rises markedly. In the high and moderate risk regions of Columbia and Peru, for example, the risk of malaria doubled when spraying ceased in the 1990s. The disease has returned to areas in which it had been eradicated: urban areas of the Amazon Basin, Korea, Armenia, Azerbaijan, and Tajikistan. In Sri Lanka, malaria cases fell from 2.8 million and 7,300 deaths per year before DDT spraying, to 17 cases and no deaths (Roberts, Manguin, and Mouchet 2000). When the spraying stopped in 1961, malaria jumped back to 500,000 cases by 1969 (Attaran et al. 2000, 729). The spread of the disease means that vectors were still available.

Also their toxic effects are not curable e.g. effects on the nervous system, liver, kidneys, and immune systems; reproductive effects where it's said to have negative reproductive effects; mutagenic effects; teratogeniceffects; Carcinogenic effects; and its Ecological effects such as effects on aquatic organisms environments and birds.

\section{WHAT'S THE ALTERNATIVE?}

As we have seen the risk associated with the use of DDT in malaria vector control, the following alternative have found to work in various areas around the world Taking an example of Mexico where they managed to eradicate malaria through community involvement in control strategies, improved surveillance and treatment i.e. preventive medication of all people who have had malaria the year or two before, and the use of new household spraying techniques i.e. pyrethroid-based insecticide that unlike DDT, does not persist in the environment, Community actions also involved wading into water near their villages every two weeks to remove algae by getting rid of the plants; the villagers deprive the mosquito larva of their habitat and population of the insect subsequently declined. These strategies were undertaken in areas with high malaria risk.

Mexico has dramatically reduced malaria transmission. In 2001 there were just 4,996 cases of malaria -- down from 15,121 in 1998. Significantly, this decrease came about without a drop of DDT. 
Similarly in Kenya instead of DDT alternatives are the use of larvicide Bacillus thuringiensis israelensis (BTi) and its related products. Also mentioned are other strategies as alternatives to DDT in the control of malaria such as insecticide-treated nets (ITN), house spraying, space spraying, integrated vector management (IVM), biological control (e.g. bacteria, fungi, nematodes, copepods, larvicidal fish).

\section{REFERENCES}

1. Roger E. Meiners and Andrew P. Morriss (2001), the Legacy of the DDT Ban; DDT's Legacy: Malaria's Return PERC Reports: Volume 19, No.2, June 2001.

2. Grasshopper effect and tracking Hazardous Air pollutants : in science and Environment bulletinmay/June 1998 retrieved from www.ec.gc.ca on 29th November 2008

3. The international POPS elimination Project January 2006Approaches to Effective Malaria Control that Avoid DDT in Kenya: Use of Bacillus elethuringiensis israelensis retrieved from www.ipen.org on 23rd February 2009

4. WHO (2005), Frequently asked questions on DDT use for disease vector control

5. IDRC Case study Mexico (malaria), fighting malaria without DDT, Better management of environments a key factor to disease control

6. Attaran, Amir, Donald R. Roberts, Chris F. Curtis, and Wenceslaus L. Kilama. 2000 Balancing Risks on the Backs of the Poor. Nature Medicine 6(7): 729Đ31

7. Roberts, D. R., S. Manguin, and J. Mouchet. 2000. DDT House Spraying and Re-emerging Malaria. Lancet 356: 330Đ32. 